\title{
Diseño de un incinerador de lecho fluidizado para procesar desechos sólidos orgánicos
}

\section{(Design of a fluidized bed incinerator to process organic solid waste)}

\author{
Salvatore La Verde Spano ${ }^{1}$, Leonardo Taylhardat ${ }^{1}$
}

\begin{abstract}
Resumen:
El trabajo contempla el diseño teórico de un incinerador de lecho fluidizado para la eliminación de desechos orgánicos, según los parámetros más avanzados publicados en los trabajos sobre el tema, tomando en consideración las normativas previstas para este tipo de equipo y relacionadas con el ambiente. Los diseños actuales de los incineradores y gasificadores de lecho fluidizado demostraron ser principalmente de forma cilíndrica. Los elementos de diseño a los cuales se les atribuye mayor importancia son la temperatura de operación del incinerador y el tiempo de residencia del desecho dentro del mismo. La temperatura de operación del incinerador debe estar entre 850 y $1.100^{\circ} \mathrm{C}$ para asegurar la destrucción casi completa de los compuestos orgánicos en el desecho y evitar la emisión de contaminantes, principalmente dioxinas y furanos, el tiempo de residencia es de tres (3) minutos, y se plantea el uso de arena de sílice como material de soporte para cumplir con las normativas ambientales, técnicas y de salubridad. Las dimensiones del incinerador para una capacidad de tratamiento de $1 \mathrm{t} \mathrm{hr}^{-1} \mathrm{se}$ determinaron en función del tiempo de residencia, volumen y densidad del desecho a incinerar, y fueron $514 \mathrm{~mm}$ de diámetro y $6.650 \mathrm{~mm}$ de alto.
\end{abstract}

Palabras clave: incinerador, fluidización, lecho, temperatura, tiempo.

\begin{abstract}
:
This work includes the theoretical design of a fluidized bed incinerator for organic waste disposal, according with the most advanced parameters published about the subject, taking into account the regulations provided for this type of equipment and related environment. Current designs of incinerators and fluidized bed gasifiers proved to be mostly cylindrical. The design element to which greater importance is ascribed to the incinerator operating temperature and residence time of the waste inside it. The incinerator operating temperature should be between 850 and $1.100^{\circ} \mathrm{C}$ to ensure almost complete destruction of the organic compounds in the waste and prevent the emission of pollutants, mainly dioxins and furans, the residence time is three (3) minutes and silica sand is proposed to be used as support material, to comply with environmental regulations and health techniques. Incinerator dimensions for 1 ton hr-1 treatment capacity are determined by the residence time, volume and density of the waste to be incinerated, and were $514 \mathrm{~mm}$ in diameter and $6.650 \mathrm{~mm}$ of high.
\end{abstract}

Keywords: incinerator, fluidization bed, temperature, time.

\footnotetext{
${ }^{1}$ Universidad Central de Venezuela, Maracay-Venezuela ( \{salvatore.laverde, leonardo.taylhardat\}@ucv.ve)
} 


\section{Introducción}

El desarrollo actual de la humanidad y el crecimiento poblacional han traído consigo una gran producción de residuos, los cuales tienen un impacto negativo tanto en el ambiente como en la salud. Su acumulación y la búsqueda de nuevos sitios de rellenos sanitarios se hace complicado, por las exigencias ambientales inherentes debido tanto a su gran volumen, como a los costos generados por su procesamiento, además, los vertederos se han convertido en focos permanentes de contaminación y por esta razón el manejo de los desechos sólidos ha devenido en un problema de salud pública, la Organización para la Cooperación y Desarrollo Económico y la Organización Panamericana para la Salud estiman que se producen en la actualidad de 0,8 a 1,4 kg de desechos sólidos totales por habitante-día (Organization for Economic Co-operation and Development, 2005; Organización Panamericana de la Salud, 2005), y tomando en cuenta que la población mundial para el año 2011 era de 6.974 millones de personas, se tiene que ese año se produjeron cerca de 9.764.000 toneladas de desechos (Fondo de Población de las Naciones Unidas, 2011).

En Venezuela el procesamiento de desechos sólidos en general es limitado, por cuanto existen innumerables vertederos de basura a cielo abierto en las principales ciudades y pocas alternativas adecuadas de manejo, como por ejemplo los rellenos sanitarios, se tiene que para el año 2010, solo se contaba con dos de estos en el país, esto trae como consecuencia que se puedan presentar problemas de contaminación de cuerpos de agua por lixiviación de contaminantes.

La Facultad de Agronomía de la Universidad Central de Venezuela (FAGRO), con sede en Maracay, Venezuela, no escapa a esta realidad debido a su vasta extensión (180 hectáreas, de las cuales 17 hectáreas son de jardines y áreas verdes), en donde se genera una gran cantidad de desechos sólidos orgánicos, tales como desechos y residuos vegetales, papelería no reciclable, y en donde además hay un comedor universitario que genera desechos sólidos.

Una posible solución al manejo y tratamiento de los residuos lo constituye la incineración. En las últimas décadas, una gran cantidad de países industrializados la han empleado como tratamiento de los residuos sólidos urbanos. La utilización de esta tecnología permite reducir en gran medida el peso (75\%) y el volumen (90\%) de los residuos a tratar y además, obtener energía. En virtud del alto poder calorífico requerido para incinerar el material y del potencial contaminante de las emisiones, los sistemas de incineración han evolucionado hacia tecnologías capaces de alcanzar mayores rendimientos en la combustión y mayor eficacia en la eliminación de contaminantes (Romero, 2001).

Uno de los avances tecnológicos que viene desarrollándose es la denominada incineración de lecho fluidizado, la cual se caracteriza por realizar la combustión no directamente en el desecho sino utilizando un material inerte como vehículo. Este material inerte usualmente está constituido 
por: arenas, cenizas, piedra caliza u otro material, al cual se le agrega el desecho a ser incinerado que puede ser: combustible, carbón, material vegetal, basura, entre otros, este se mezcla mediante un sistema neumático al material inerte y se procede a la combustión controlada del mismo, se recomienda que el material combustible no sea mayor al 5\% del peso total del lecho, usualmente el rango varía de 2 al $5 \%$ del mismo.

El incinerador de lecho fluidizado no solo elimina los desechos orgánicos sino que evita la conversión de dichos desechos en metano $\left(\mathrm{CH}_{4}\right)$, como cuando estos son acumulados en los rellenos sanitarios y como el metano equivale a 21 veces el efecto del Dióxido de Carbono $\left(\mathrm{CO}_{2}\right)$ al efecto invernadero en la atmosfera se reduce el impacto sobre el mismo, obteniéndose un tratamiento más eficiente. (International Carbon Bank \& Exchange, 2000; Tryger Ltda, (s. f.))

El material a incinerar es muy variado, desde residuos sólidos municipales (RSM) usados en los incineradores de López y Bautista (2004) y Reis et al. (2007), lodos residuales de plantas de tratamiento de agua en el incinerador de Von Roll Inova en Balogh y Nollet (2008), hasta carbón y biomasa usado en gasificadores e incineradores estudiados en Velez et al. (2009) y Pöykiö et al. (2009).

El presente trabajo constituye una propuesta factible desde el punto de vista técnico para eliminar la gran cantidad de desechos sólidos orgánicos generados en la Facultad de Agronomía, teniendo como objetivo diseñar un incinerador de lecho fluidizado para procesar desechos sólidos orgánicos agrícolas e inorgánicos combustibles procedentes de FAGRO.

\section{Materiales y métodos}

Las dimensiones del incinerador dependen de la cantidad de desechos a incinerar y de la frecuencia de generación (flujo másico). La altura mínima de la cámara de combustión se calcula a partir de la sección transversal seleccionada para el incinerador.

Dado que el lecho se va a expandir al fluidizarse, y que dicha expansión puede llegar al 100\% o más del lecho en reposo, la altura de diseño de la cámara de combustión se recalcula y se establece en por lo menos el doble de la altura calculada anteriormente, para evitar así el arrastre de partículas sin incinerar fuera del incinerador.

El siguiente paso es elegir y caracterizar los materiales que se usan como soporte del lecho, para ello se determina la granulometría de las partículas del soporte a utilizar, con lo cual se encuentran los diámetros mínimos y máximos en milímetros.

Se selecciona el equipo adecuado que garantice el suministro del aire necesario para fluidizar el lecho, para ello se calcula el caudal necesario a través de la ecuación.

$$
Q=v A
$$


Donde $A$ es la sección transversal del incinerador y $v$ es la velocidad mínima de fluidización, la cual se calcula teóricamente según la fórmula determinada por Coltters y Rivas (2004).

$$
v_{m f}=\left(9,7119 \times 10^{-7}\right) X^{(0,84268 \pm 0,01601)}
$$

para un diámetro de partícula entre $95 \mu \mathrm{m} \leq D_{p} \leq 800 \mu \mathrm{m}$, donde $X$ es una constante y se calcula con la siguiente formula:

$$
X=\frac{D_{P}^{2}\left(\rho_{S}-\rho_{g}\right) g}{\mu}\left(\frac{\rho_{S}}{\rho_{g}}\right)^{1,23}
$$

Donde $\rho_{s}$ es la densidad de la arena o material del lecho, $\rho_{g}$ es la densidad del fluido (aire a 25 $\left.{ }^{\circ} \mathrm{C}\right), g$ la aceleración de la gravedad, $D_{p}$ el diámetro de la arena y $\mu$ la viscosidad del fluido.

Se debe trabajar en el diseño con velocidades mínimas de fluidización y por lo tanto se obtienen caudales mínimos, al seleccionar el equipo es necesario tomar en cuenta este factor y escoger aquel que entregue un caudal por encima de tales valores.

Otros de los factores a tomar en cuenta para lograr una combustión eficiente son las pérdidas de calor que pueden ocurrir por la falta de aislamiento, por lo tanto se revisa la bibliografía para determinar si dichas pérdidas afectan la eficiencia de la combustión y cómo evitarlo.

Finalmente se establece el periodo y frecuencia, y los puntos clave de toma de temperatura a lo largo del proceso, así como el análisis de los gases de la combustión a la salida del incinerador.

\section{Resultados y discusión}

\subsection{Identificación y análisis de los diseños de incineradores de lecho fluidizado}

Se eligió diseñar el incinerador con sección cilíndrica, ya que es la forma geométrica más sencilla de construir y de operar, sumado a que la distribución del lecho es más uniforme, siendo el material predominantemente usado en el diseño el acero al carbono con accesorios en acero inoxidable. Dado que la forma elegida del incinerador es cilíndrica la sección transversal libre $A$ se calcula con la ecuación del área de una sección circular.

Los elementos de diseño a los cuales se le atribuye mayor importancia son la temperatura de operación del incinerador y el tiempo de residencia del desecho dentro del mismo. Para cumplir con las normas sobre calidad del aire y control de la contaminación atmosférica (República de Venezuela, 1995), ver Tabla 1, la temperatura de operación del incinerador debe estar entre 850 y $1.100{ }^{\circ} \mathrm{C}$ y el tiempo de residencia en tres (3) minutos. 
Tabla 1. Límites de emisión de contaminantes del aire y de opacidad para fuentes fijas de contaminación atmosférica.

\begin{tabular}{|l|c|}
\hline \multicolumn{1}{|c|}{ Contaminantes } & Mg. $^{-3}$ \\
\hline Cloruro de Hidrogeno $(\mathrm{HCl})$ & 50 \\
\hline Dióxido de Azufre $\left(\mathrm{SO}_{2}\right)$ & 100 \\
\hline Fluoruro de Hidrogeno $(\mathrm{HF})$ & 2 \\
\hline Bromuro de Hidrogeno $(\mathrm{HBr})$ & 5 \\
\hline Monóxido de Carbono $(\mathrm{CO})$ & $400 \mathrm{ppm}$ \\
\hline Óxidos de Nitrógeno $(\mathrm{NOx})$ & 200 \\
\hline Partículas solidas & $200\left(\right.$ residuos $\left.1-3 \mathrm{t}^{-1} \mathrm{~h}^{-1}\right)$ \\
\hline Dioxinas y Furanos & $0,1 \mathrm{ng}^{-3} \mathrm{~m}^{-3}$ \\
\hline Hidrocarburos aromáticos policíclicos $(\mathrm{PAH})$ & 0,05 \\
\hline
\end{tabular}

Fuente: República de Venezuela, (1995).

\subsection{Diseño del incinerador para la producción de desechos sólidos de la Facultad de Agronomía}

En FAGRO se genera aproximadamente un total de $3 \mathrm{~kg} \cdot \mathrm{m}^{-2} \cdot \mathrm{mes}^{-1}$ de desechos orgánicos, y dado que el área de jardines de FAGRO es de aproximadamente 17 ha, se generan alrededor de 510 t.mes ${ }^{-1}$ de desechos orgánicos agrícolas lo que da alrededor de $17 \mathrm{t}^{-d i a^{-1}} \mathrm{y}$ por lo tanto $0,71 \mathrm{t}^{-h r^{-1}}$, se redondea este valor hasta $1 \mathrm{t}^{\mathrm{h} r^{-1}}$ tomando en cuenta las distintas variables que inciden en el crecimiento de las plantas y caídas de hojas y ramas.

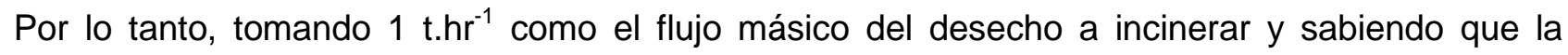
densidad del desecho agrícola es de 0,09042 t.m-3 (determinado experimentalmente por los autores en el Laboratorio de Materiales de FAGRO) y tres minutos de tiempo de residencia, se determinó el volumen de desecho a incinerar el cual equivale al volumen de la cámara de combustión, siendo $0,553 \mathrm{~m}^{3}$.

Dado que el incinerador de lecho fluidizado semeja una tubería, se calcula el diámetro libre, a partir de las tuberías de acero sin costura disponibles en el mercado, se recomienda que este diámetro no sea muy grande debido a que se requerirá mucha energía para fluidizar el lecho, en este trabajo, se fijó en 0,514 m, el cual es el diámetro interno de una tubería de 30 pulgadas (762 $\mathrm{mm}$ ) de acero al carbono sin costura, ya que se consigue fácilmente en el mercado nacional, menos dos veces el alto de un ladrillo refractario $(0,1143 \mathrm{~m})$.

$$
0,762 m-2 \times 0,00953 m-2 \times 0,1143 m=0,514 m
$$

Para el diámetro de $0,514 \mathrm{~m}$ corresponden un área transversal circular libre $A$ de $0,208 \mathrm{~m}^{2}$ y una altura mínima de 2,66 m.

Dado que el lecho se expandirá hasta un 100\% o más de la longitud del lecho en reposo, se recalcula la altura del mismo con un factor de seguridad de 2,5 y se establece en $6.650 \mathrm{~mm}$ para evitar el arrastre de partículas sin incinerar fuera del incinerador. 
Se analizaron tres tipos diferentes de arena en el laboratorio de materiales de FAGRO: arena de sílice, arena de los médanos de Coro y arena lavada empleada en construcción, y se obtuvo que la más adecuada es la arena de sílice, ya que se busca obtener un diámetro promedio entre 0,3 $\mathrm{mm}$ y $0,6 \mathrm{~mm}$ y esa fue la arena que proporcionó la caracterización más uniforme. Se determinó la densidad de la arena, obteniéndose un valor de 1.528,2 kg. ${ }^{-3}$, ver Tabla 2.

Tabla 2. Valores de densidad y diámetro de la arena del lecho.

\begin{tabular}{|l|c|}
\hline Densidad de la arena $\rho_{s}$ & $1.528,2 \mathrm{~kg} \cdot \mathrm{m}^{-3}$ \\
\hline Diámetro de la partícula $\mathrm{D}_{\mathrm{p}}$ & $>0,3 \mathrm{~mm}$ \\
& $<0,6 \mathrm{~mm}$ \\
\hline
\end{tabular}

En la fabricación del incinerador se empleará como materia prima acero al carbono por su maleabilidad para trabajarla, su precio accesible y su facilidad para adquirirla.

El plato distribuidor que soportará el lecho será de acero inoxidable de $10 \mathrm{~mm}$ de espesor y con múltiples perforaciones. Para evitar que la arena se vaya por las perforaciones cuando el incinerador esté en reposo, se colocan en cada perforación toberas de acero inoxidable.

El aire para fluidizar el lecho será suministrado por un compresor debido a que este garantiza el flujo constante de aire y mantiene la presión.

Para obtener el caudal con la fórmula (1), primero se calcula la velocidad mínima de fluidización con las fórmulas (2) y (3) con los datos de la Tabla 3, (Potter y Wiggert, 1998) y la aceleración de gravedad $g$ como 9,81 m.s. ${ }^{-2}$.

Tabla 3. Propiedades del aire a $25^{\circ} \mathrm{C}$.

\begin{tabular}{|l|c|}
\hline Densidad $\rho_{g}$ & $1,18 \mathrm{~kg} \cdot \mathrm{m}^{-3}$ \\
\hline Viscosidad $\mu$ & $1,783 \times 10^{-5} \mathrm{~kg} \cdot \mathrm{m}^{-1} \cdot \mathrm{s}^{-1}$ \\
\hline
\end{tabular}

Se utilizan los valores extremos de los diámetros de la arena y se obtiene un rango de velocidades mínimas de fluidización que va desde $225 \mathrm{~m} \cdot \mathrm{h}^{-1}$ hasta $720 \mathrm{~m} \cdot \mathrm{h}^{-1}$, las cuales están dentro del rango de velocidades mencionadas por Prieto (s. f.), con la velocidad mínima de fluidización y el área transversal del incinerador $0,208 \mathrm{~m}^{2}$ se calcula el caudal con la ecuación (1), obteniéndose un rango desde $46,8 \mathrm{~m}^{3} \cdot \mathrm{h}^{-1}$ hasta $149,8 \mathrm{~m}^{3} \cdot \mathrm{h}^{-1}$, para seleccionar el compresor se transforman las unidades a litros por minuto y se obtiene un rango de $780 \mathrm{lpm}$ hasta $2.500 \mathrm{lpm}$, el cual puede ser alcanzado con un compresor de $25 \mathrm{HP}$, cuatro pistones en "V", doble etapa de compresión, 2.800 Ipm y 175 psi.

El cuerpo del incinerador mostrado en la Figura 1 es de acero al carbono, se fabrica a partir de un tubo de acero sin costura de $762 \mathrm{~mm}$ y consta de siete (7) secciones, la primera sección es de $500 \mathrm{~mm}$ de altura, las otras seis (6) de $1.143 \mathrm{~mm}$ de altura cada una para así aprovechar la longitud del ladrillo refractario, obteniéndose una altura total de $7.538 \mathrm{~mm}$ para garantizar la altura necesaria de $6.650 \mathrm{~mm}$ de la cámara de combustión; para unir cada sección se coloca un flange o 
brida de $100 \mathrm{~mm}$ de ancho y $12 \mathrm{~mm}$ de espesor con perforaciones de 14,29 $\mathrm{mm}$ y conectar con pernos de 12,7 mm. En cada sección del incinerador, se encuentran orificios con tapón macho de $12,7 \mathrm{~mm}$ de diámetro donde se enroscan los termopares para la toma de temperaturas, y otros dos orificios para los indicadores de caída de presión ubicados en la sección del lecho. La primera sección de abajo hacia arriba del incinerador es la de entrada de aire para fluidizar el lecho proveniente del compresor y el combustible. Entre esta sección y la siguiente se encuentra el plato distribuidor, el mismo es de acero inoxidable con un espesor de $10 \mathrm{~mm}$, en dicho plato van ubicadas las toberas de aire de fluidización.

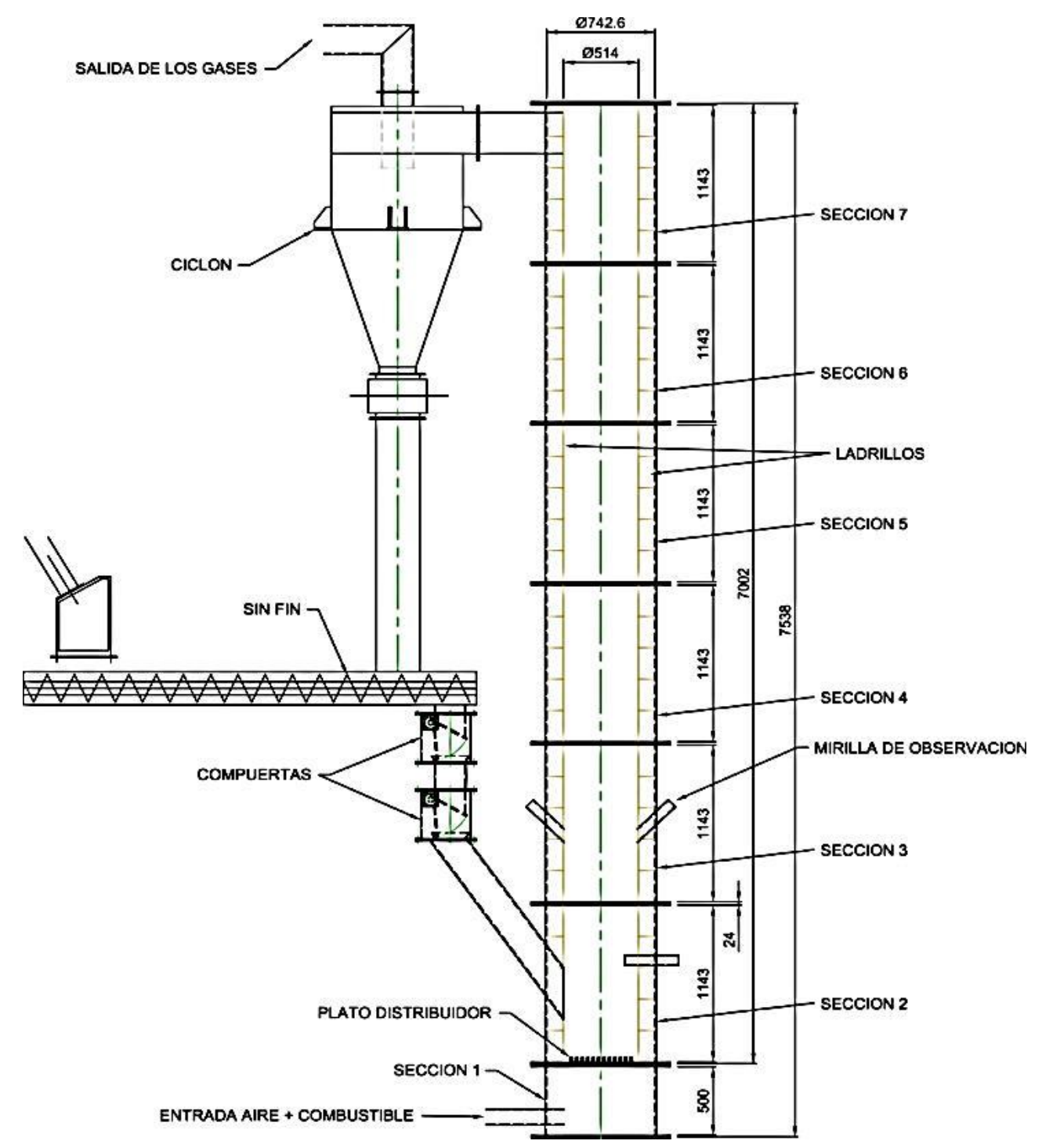

Figura 1. Esquema del Incinerador de Lecho Fluidizado e indicaciones de partes componentes.

Las toberas se componen de un tubo sin costura de acero inoxidable AISI-304L de 9,525 mm (0,375 pulgada) de diámetro y $56 \mathrm{~mm}$ de longitud, de los cuales $44 \mathrm{~mm}$ sobresalen del plato distribuidor al que están soldados, el extremo superior está cerrado y a $7 \mathrm{~mm}$ de este se localizan 4 orificios equidistantes de $3,175 \mathrm{~mm}$ (1/8 de pulgada), ver Figura 2. 


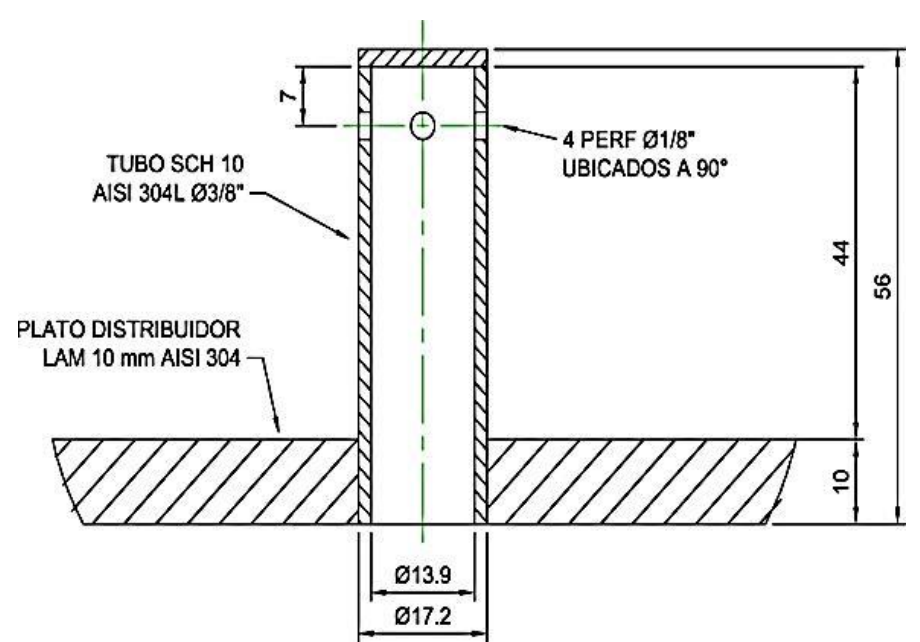

Figura 2. Detalle Tobera.

La segunda sección es la sección del lecho, tiene un ducto de entrada de desechos con un ángulo de $55^{\circ}$ hacia la pared del mismo para garantizar que todo el material a incinerar deslice y entre al incinerador, dicho ducto posee dos compuertas de seguridad para dosificar la entrada de material al incinerador, dichas compuertas se activarán cada tres minutos (tiempo de residencia) y el desecho se lleva al incinerador a través de un transportador tipo sin fin con un caudal de 1 t. $\mathrm{h}^{-1}$.

En la tercera sección del incinerador hay dos entradas laterales opuestas en forma de "V" con un ángulo de $45^{\circ}$ hacia la pared del mismo, fabricadas de tubos de acero sin costura de 63,5 mm (2 $1 / 2$ pulgadas) de diámetro, estas se usan como mirilla de observación y para introducir el soplete de soldadura de oxígeno y acetileno para el arranque del sistema.

En la última sección se coloca una tubería cuadrada lateralmente al incinerador para conectarlo a un ciclón que recoge las partículas de lecho y de materia orgánica sin incinerar y las vuelve a insertar dentro del incinerador en la segunda sección. En la salida del ciclón se coloca la chimenea y los rociadores de agua para enfriar el gas rápidamente a menos de $200{ }^{\circ} \mathrm{C}$ y así evitar la formación de dioxinas y furanos.

A la salida de la chimenea se colocará un analizador de gases comercial con capacidad de medición de oxígeno, dióxido de carbono, monóxido de carbono, óxidos de nitrógeno, dióxido de azufre, temperatura, presión, velocidad, caudal, exceso de aire y eficiencia de combustión.

El aislamiento térmico del incinerador se realiza tanto interna como externamente, internamente el aislamiento se logra con ladrillos refractarios de 228,6 x 114,3 x 63,5 mm los cuales se adhieren a las paredes con un pegamento especial, luego se recubrirán los ladrillos con un friso de cemento refractario y el recubrimiento externo se hará con fibra de vidrio recubierta con papel metalizado de $25,4 \mathrm{~mm}$ de espesor.

Para monitorear la temperatura dentro del incinerador a todo lo largo del proceso se usan termopares tipo $\mathrm{K}$ de acero inoxidable con intervalos de temperatura de -200 a $1.371^{\circ} \mathrm{C}$, la temperatura se registra en un panel de control. 


\subsection{Descripción de las especificaciones teóricas de operación}

El sistema de incineración trabaja según el esquema mostrado en la Figura 3, en la cual se observa que el mismo consta de las siguientes etapas: inyección de aire y encendido del sistema, carga de los desechos e incineración y post combustión y análisis de los gases.
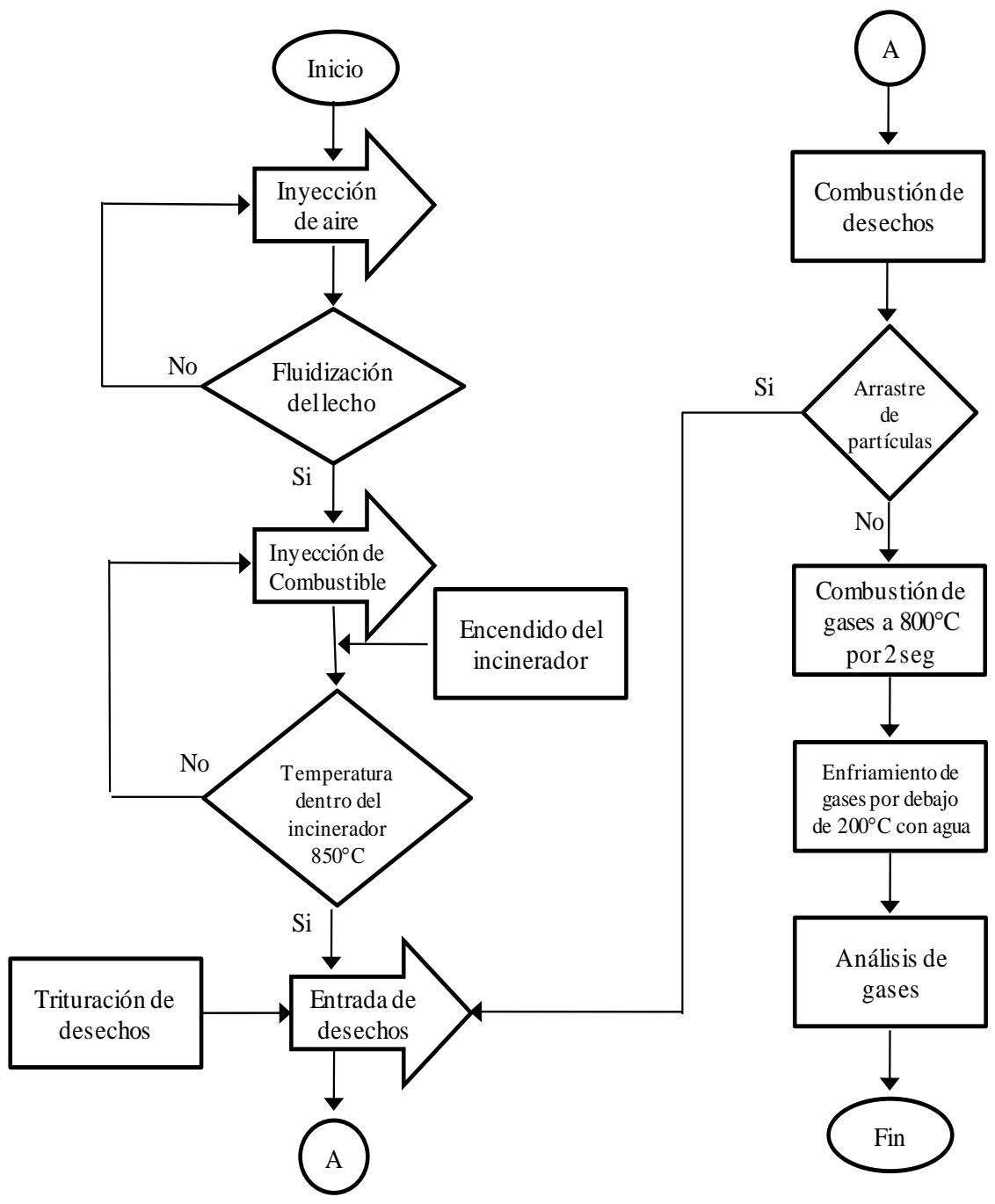

Figura 3. Flujograma de la operación del incinerador de lecho fluidizado.

El material de soporte o de lecho seleccionado es arena, debido a que es fácil de fluidizar, es estable y conserva sus características a altas temperaturas y la operación se realiza bajo condiciones específicas de altura estática del lecho de arena de 0,15 a 0,20, y tamaño promedio de la partícula de arena entre 0,3 y $0,6 \mathrm{~mm}$ y con flujo de gas y aire constante, tal como se indica en el Tabla 4.

Tabla 4. Especificaciones de operación definidas para el diseño del incinerador de FAGRO para 1 t.hr ${ }^{-1}$.

\begin{tabular}{|l|c|}
\hline Temperatura de operación $\left({ }^{\circ} \mathrm{C}\right)$ & $850-1.100$ \\
\hline Tiempo de residencia $(\mathrm{min})$ & 3 \\
\hline Altura estática de lecho $(\mathrm{m})$ & $0,15-0,20$ \\
\hline Exceso de aire $(\%)$ & $5-10$ \\
\hline
\end{tabular}




\subsubsection{Inyección de aire}

El arranque de la operación del sistema se realiza mediante la inyección de aire para fluidizar el lecho, una vez fluidizado se hace pasar el combustible y se enciende mediante un generador de chispas cerámico. Una vez que el lecho de arena alcanza la temperatura de $850{ }^{\circ} \mathrm{C}$, los desechos se incorporan al incinerador a través de un transportador sin fin por una ventana de alimentación en la parte lateral del mismo. La introducción de los desechos será dosificada por dos compuertas para evitar que los desechos se enciendan antes de entrar al incinerador y generar un efecto en cadena quemándose el transportador sin fin.

\subsubsection{Combustión de desechos}

Después de mezclarse las partículas volátiles, éstas se queman en lo que se conoce como combustión homogénea o combustión de fase gas. El material residual se queda en el lecho y su combustión, por lo general, involucra tres procesos: a) intercambio de gas entre las burbujas y la fase de partículas (que depende del tamaño de la burbuja), b) difusión de los gases que forman parte de la reacción hacia y desde la superficie de la partícula, la cual depende del tamaño de la misma, y c) reacción química en la superficie de la partícula, y que están en función de la temperatura del sistema.

\subsubsection{Toma de datos}

Durante la operación del incinerador se lleva a cabo un registro de los diversos parámetros de operación y fluidización (velocidad de fluidización, flujo de combustible, presión y temperatura en diversos puntos del incinerador) a través de los orificios que se realizaron en cada sección del incinerador.

\subsubsection{Combustión de gases}

Los gases productos de la combustión se hacen pasar por un ciclón donde por gravedad se recogen los desechos sólidos sin incinerar y la arena arrastrada (en el supuesto de que llegara a ocurrir), por otro lado los gases de la combustión deben pasar por una segunda cámara de combustión con un tiempo de residencia de al menos dos segundos a una temperatura mínima de $850{ }^{\circ} \mathrm{C}$ (Environmental Protection Agency,1992) con la finalidad de reducir la emisión de contaminantes. Posteriormente los gases antes de salir a la atmosfera son enfriados en la chimenea por debajo de los $200{ }^{\circ} \mathrm{C}$ con chorros de agua para evitar la formación de dioxinas y furanos. Adicionalmente se analizan los gases de la combustión $\left(\mathrm{CO}, \mathrm{SO}_{2}, \mathrm{NO}_{\mathrm{x}}, \mathrm{O}_{2}\right)$ a la salida de la chimenea con un analizador de gases comercial. 


\section{Discusión}

Se seleccionó la forma cilíndrica ya que es la más utilizada y recomendada para el diseño del incinerador de lecho fluidizado en la literatura revisada (López y Bautista, 2001; Vélez et al., 2009), con la notable excepción de Zhang et al., (2009) quienes utilizaron un dispositivo rectangular; de igual manera la elección de acero al carbono y acero inoxidable como materiales para la construcción obedece a que son los más frecuentemente utilizados y recomendados en la literatura porque resisten adecuadamente las altas temperaturas de operación del sistema (López y Bautista, 2001; Vélez et al., 2009).

La temperatura de operación del incinerador debe estar entre 850 y $1.100{ }^{\circ} \mathrm{C}$, ya que con ello se asegura la destrucción casi completa de los compuestos orgánicos en el desecho (Air \& Waste Management Association; 1992), por otra parte, el tiempo de residencia es de tres (3) minutos según lo establece Prieto (s. f.) ya que con ese valor de tiempo aumenta la probabilidad de que tengan lugar las reacciones de desulfuración, y que cumplan con los límites de emisiones máximos mostrados en la Tabla 1.

El diseño del plato distribuidor se realizó considerando las investigaciones de (López y Bautista, 2004; Vélez et al., 2009; Zhang et al., 2009; Bautista et al., 2004; López et al., 2008; Gogebakan y Selçuk, 2009), quienes indicaron que este diseño ha resultado adecuado y eficiente.

Dado que el aire para fluidizar el lecho será suministrado por un compresor siguiendo la recomendación de López y Bautista (2004), y como el diseño se realizó basado en velocidades mínimas y por lo tanto, en caudales mínimos de operación, al seleccionar el compresor es necesario tomar en cuenta este factor y seleccionarlo de tal forma que entregue un caudal por encima de este valor de diseño, además que se necesita añadir más aire para cumplir con el exceso de aire de 5 a 10\% necesario para alcanzar la oxidación óptima del combustible y evitar la formación de $\mathrm{CO}$, ya que el control de la combustión y la reducción de las emisiones a la atmósfera en un incinerador de lecho fluidizado dependen de factores como la materia prima, el material de soporte, y los mecanismos de inyección y evacuación de los gases. Al respecto, López et al. (2008) mencionan que existe una correlación entre la temperatura, el tiempo de residencia y el grado de emisión. Hasfelriis (1987) y Wang (1993) han registrado que ciertas condiciones de operación minimizan la formación de CO y reducen la emisión de dioxinas y furanos. Por su parte, Wiley (1987) sugiere como mínimo un nivel de oxígeno de 1 a $2 \%$ en volumen, involucrando un incremento de 5 a $10 \%$ de exceso de aire al sistema, para con ello alcanzar la oxidación óptima del combustible y evitar la formación de monóxido de carbono (CO).

La razón de enfriar bruscamente el gas por debajo de $200{ }^{\circ} \mathrm{C}$ y así evitar la formación de dioxinas y furanos de novo, es que estos compuestos tienen la capacidad de formarse nuevamente cuando el gas se enfría lentamente entre 200 y $400^{\circ} \mathrm{C}$ (Romero, 2001). Es importante destacar que para el 
correcto funcionamiento del sistema el material a incinerar debe estar previamente desmenuzado, tal como lo sugieren Lopez et al. (2008)

La importancia del aislamiento térmico obedece al hecho de evitar o minimizar las pérdidas de calor, las cuales afectan la eficiencia de la combustión (López y Bautista, 2004; Bautista et al., 2004; López et al., 2008), si bien los autores mencionados solo calculan la eficiencia de la combustión, López et al. (2008), pero no realizan cálculos de pérdidas, aun así recomiendan el aislamiento para mejorar la eficiencia.

Se utilizó el soporte de lecho de arena basado en las investigaciones de López y Bautista (2004), Bautista et al. (2004), López et al. (2005), López et al. (2008), Gogebakan y Selçuk (2009), Zhang et al. (2009), y por la compañía suiza Von Roll Inova (Balogh y Nollet, 2008), quienes lo recomiendan por su facilidad para fluidizar, estabilidad y resistencia a las altas temperaturas, asimismo, el diseño del lecho de arena se realizó siguiendo las recomendaciones de Prieto (s. f.) y López y Bautista (2004).

\section{Conclusiones y recomendaciones}

El incinerador de lecho fluidizado constituye una alternativa para el manejo de residuos de fácil construcción, manejo y mantenimiento, ya que con el uso del incinerador se consigue una reducción muy alta de los desechos sólidos ( $70 \%$ en peso y hasta $90 \%$ en volumen), con una baja emisión de gases de la combustión $\left(\mathrm{CO}, \mathrm{SO}_{2}, \mathrm{NO}_{x}, \mathrm{O}_{2}\right)$ y evitando la liberación de dioxinas y furanos.

El material de soporte para el lecho más adecuado, resultó ser la arena de sílice, aunque es posible que dependiendo de la disponibilidad, puedan utilizarse otras arenas, cenizas, piedra caliza entre otros materiales, siempre que cumplan con los requerimientos de diseño. Los materiales utilizados para su construcción pueden adquirirse fácilmente en el mercado local, lo cual redunda positivamente en la facilidad y costo de construcción del incinerador.

Si bien el incinerador del presente trabajo se diseñó para residuos orgánicos, principalmente vegetales, el mismo podría utilizarse para casi todo tipo de residuos, y aunque las dimensiones del incinerador diseñado en el presente trabajo (514 mm de diámetro y $6.650 \mathrm{~mm}$ de alto), permitirán una eliminación adecuada de $1 \mathrm{t} . \mathrm{hr}^{-1}$ de los residuos que se generan en FAGRO de la Universidad Central de Venezuela, localizada en Maracay, estado Aragua, Venezuela, este diseño puede ser fácilmente modificado y adaptado para otros valores de flujo másico de residuos distintos al de diseño ( 1 t.hr $^{-1}$ ) aplicando las ecuaciones y los procedimientos utilizados en el presente trabajo, empleando un rango de velocidades mínimas de fluidización que va desde $225 \mathrm{~m} \cdot \mathrm{h}^{-1}$ hasta 720 $\mathrm{m} \cdot \mathrm{h}^{-1}$. 


\section{Bibliografía}

Air \& Waste Management Association. (1992). Air pollution engineering manual. New York: Van Nostrand Reinhold.

Balogh, S. J., y Nollet, Y. H. (2008). Mercury mass balance at a wastewater treatment plant employing sludge incineration with offgas mercury control. Science of the total environment. $389(1), 125-131$.

Bautista Margulis, R. G., López Ocaña, G., y Torres Balcázar C. A. (2004). Tratamiento térmico de residuos sólidos municipales en un combustor experimental de lecho fluidizado. Trabajo presentado en Semana de Divulgación y Video Científico. Universidad Juárez Autónoma de Tabasco. Villahermosa, México.

Coltters, R., y Rivas, A. L. (2004). Minimum fluidation velocity correlations in particulate systems. Powder Technology. 147(1-3), 34-48.

Fondo de Población de las Naciones Unidas. (2011). Estado de la población mundial. New York, NY: UNFPA.

Gogebakan, Z., y Selçuk, N. (2009). Trace elements partitioning during co-firing biomass with lignite in a pilot-scale fluidized bed combustor. Journal of Hazardous Materials. 162(2-3); $1129-1134$.

Hasselriis, F. (1987). Optimization of combustion conditions to minimize dioxin emissions. Waste Management \& Research. 5, 311-326.

International Carbon Bank \& Exchange. (2000). Calculating greenhouse gases. Recuperado de: http://www.icbe.com/emissions/calculate.asp.

Kumar, D. (1999). Condiciones técnicas y riesgos de la incineración. Recuperado de: http://www.bvsde.paho.org/bvsacd/acodal/x.pdf.

López Ocaña, G., Bautista Margulis, R. G., Hernández Barajas, J. R., Saucedo Terán, R. A., y Rubio Arias, H. O. (2008). Combustión de residuos sólidos municipales en un sistema de lecho fluidizado experimental. Universidad y Ciencia. 24(2), 89-100.

López Ocaña, G., Bautista Margulis, R. G., y Focil Monterrubio, R. L. (2005). Combustión de lecho fluidizado aplicado al tratamiento térmico de residuos sólidos municipales generados en Villahermosa, Tabasco. Trabajo presentado en Semana de Divulgación y Video Científico. Universidad Juárez Autónoma de Tabasco. Villahermosa, México. 
López Ocaña, G., y Bautista Margulis, R. G. (2004). Diseño de un combustor experimental de lecho fluidizado para la incineración de residuos sólidos municipales. Trabajo presentado en Semana de Divulgación y Video Científico. Universidad Juárez Autónoma de Tabasco. Villahermosa, México.

Organización Panamericana de la Salud. (2005). Informe de la evaluación regional de los servicios de manejo de residuos sólidos municipales en América Latina y el Caribe. Washington, D.C.: OPS.

Organization for Economic Co-operation and Development. (2005). OECD Environmental data: compendium 2004. París: OECD Publishing.

Potter, M. C., y Wiggert, D. C. (1998). Mecánica de fluidos. México: Prentice Hall.

Pöykiö, R., Rönkkömäki, H., Nurmesniemi, H., Perämäki, P., Popov, K., Välimäki, I., y Tuomi T. (2009). Chemical and physical properties of cyclone fly ash from the grate-fired boiler incinerating forest residues at a small municipal district heating plant (6MW). Journal of Hazardous Materials. 162(2-3), 1059-1064.

Prieto, I. (s. f.). Centrales térmicas. Sistemas de combustión de lecho fluido. Recuperado de: http://ocw.uniovi.es/pluginfile.php/1011/mod_resource/content/1/1C_C12757_0910/04_GT13 _Centrales_termicas_de_lecho_fluido.pdf.

Reis, M. F., Sampaio, C., Brantes, A., Aniceto, P., Melim, M., Cardoso, L., Gabriel, C., Simão, F.; y Pereira Miguel, J. (2007). Human exposure to heavy metals in the vicinity of Portuguese solid waste incinerators. Biomonitoring of $\mathrm{Pb}, \mathrm{Cd}$ and $\mathrm{Hg}$ in blood of the general population. International Journal of Hygiene and Environmental Health. 210(3-4), 439-446.

Romero Salvador, A. (2001). Incineración de residuos sólidos urbanos. Recuperado de: http://www.icp.csic.es/cyted/Monografias/Monografias1998/C2-315.pdf.

Tryger Ltda. (s. f.). Bonos de carbono. Recuperado de: http://www.trygerltda.cl/.

USA. Environmental Protection Agency. (1992). Control techniques for volatile organic emissions from stationary sources. Report EPA 453/R-92-018. Research Triangle Park, NC: Environmental Protection Agency.

Vélez, J. F., Chejne, F., Valdés, C. F., Emery, E. J., y Londoño, C. A. (2009). Co-gasification of Colombian coal and biomass in fluidized bed: An experimental study. Fuel. 88(3), 424-430. 
Venezuela. República de Venezuela. (1995). Gaceta Oficial de la República de Venezuela, $N^{\circ}$ 4899. (Extraordinaria). Decreto $N^{\circ} 638$ de 26 de abril de 1995. Normas sobre calidad del aire y control de la contaminación atmosférica. Caracas: República de Venezuela.

Wang, W. (1993). Emission control hardware cost in California. Report 80144. Toronto: Ontario Hydro.

Wiley, S. K. (1987). Incinerate your hazardous waste. Hydrocarbon Processing. 66(6); 51-54.

Zhang, Y., Jin, B., y Zhong, W. (2009). Experimental investigation on mixing and segregation behavior of biomass particle in fluidized bed. Chemical Engineering and Processing: Process Intensification. 48(3), 745-754. 